\title{
Number of Mediastinal Lymph Nodes as a Prognostic Factor in PN2 Non Small Cell Lung Cancer: A Single Centre Experience and Review of the Literature
}

\author{
Silvia Takanen*, Caterina Bangrazi, Vanessa Graziano,Alessandro Parisi, Blerina \\ Resuli, Luca Simione, Rossella Caiazzo, Nicola Raffetto, Vincenzo Tombolini
}

\begin{abstract}
Currently the most important prognostic factor in lung cancer is the stage. In the current lung TNM classification system, $\mathrm{N}$ category is defined exclusively by anatomic nodal location though, in other type of tumours, number of lymph nodes is confirmed to be a fundamental prognostic factor. Therefore we evaluated the number of mediastinal lymph nodes as a prognostic factor in locally advanced NSCLC after multimodality treatment, observing a significant effect of the number of lymph nodes in terms of OS $(p<0.01)$ and DFS (p<0.001): patients with a low number of positive mediastinal nodes have a better prognosis.
\end{abstract}

Keywords: Locally advanced NSCLC - number of lymph nodes - prognosis

Asian Pac J Cancer Prev, 15 (18), 7559-7562

\section{Introduction}

Lung cancer is one of the most common cause of cancer-related death for men and women in the world. The prevalent histology is non-small cell lung carcinoma (NSCLC), including squamous cell carcinoma, adenocarcinoma and large cell carcinoma (Moretti et al., 2009 ), with a 5- year survival rate of $67 \%$ (stage IA) to $<5 \%$ (stage IV) (End, 2006).

Currently the most important predictor of survival in lung cancer is the stage (TNM) (Kligerman and Abbot, 2010; Goldstraw, 2009). Despite the new staging system (Kligerman and Abbot, 2010), stage III NSCLC remains a very heterogeneous disease, with a 5-year survival rate ranging from $35 \%$ to $5 \%$. There are two major treatment targets for of patients with stage III : locoregional control and control of micrometastases, preventing distant metastatic disease (Penland et al., 2004; Bradley et al., 2005). The standard of care is represented by multimodality treatment, comprehending surgery for resectable disease, perioperative chemotherapy and radiation therapy (RT) for patients with pathological (p) N2 disease (Okamoto, 2008).

In current TNM classification system, $\mathrm{N}$ category is defined exclusively by anatomic nodal location, though number of lymph nodes confirmed to be a fundamental prognostic factor as in other type of tumours.

In our study, we assessed, in patients with stage III N2 category NSCLC disease, the prognostic value of the number of lymph nodes after multimodality treatment.

\section{Materials and Methods}

We evaluated 65 patients affected by NSCLC, from January 2005 to December 2010, at the "Sapienza" University of Rome, Policlinico Umberto I, Department of Radiation Oncology.

Clinical records and official pathological reports of patients who underwent surgical resection for primary lung cancer were reviewed retrospectively.

Forty-nine patients were male $(75.4 \%)$ and 16 female (24.6\%). The mean age was 67.13 years (range 29-86). All patients underwent surgery and were completely resected: 60 lobectomy and 5 pneumonectomy.

Forty-one patients $(63 \% \%)$ were classified as IIIA stage and 24 patients (37\%) as IIIB. Pathologic staging was carried out according to the current $7^{\text {th }}$ edition of TNM classification. Histological examination showed pathological lymph nodes classified as pN2.

Histology evidenced squamous cell carcinoma in 19 patients $(30 \%)$, adenocarcinoma in 46 patients $(70 \%)$. Mediastinal nodal number evaluation were performed after surgery: all patients were divided in three groups based on the number of mediastinal lymph nodes (pN2) involved: $<5,5-7$ and $>7$ or more.

Platinum-based chemotherapy (for almost 3 cycles) was administered in all patients as adjuvant treatment.

Postoperative radiotherapy (PORT) was delivered with a 6 MV linear accelerator, median total dose of 50.4 Gy (daily fraction: 180/200cGy), using a three-dimensional external conformal radiotherapy (3D-ECRT) technique. 


\section{Silvia Takanen et al}

Target volumes included the bronchial stump, ipsilateral hilum and all mediastinal regions with pathologically involved lymph nodes.

The analysis of survival was conducted according to the Kaplan-Meier method and using the Log-Rank Test analysed overall survival (OS) and disease-free survival (DFS) for each group according to $\mathrm{T}$ and $\mathrm{N}$ stage.

\section{Results}

Median follow-up time was 30 months (range 2-76 months).

For overall patients, median OS was 36.55 months (Figure 1) and median DFS 27.99 months (Figure 2).

OS and DFS were $93.85 \%$ and $83.08 \%$ respectively at 12 months, $78.46 \%$ and $58.46 \%$ respectively at 24 months, $53.60 \%$ and $38.20 \%$ respectively at 36 months, $22,90 \%$ and $9.76 \%$ respectively at 60 months.

Local lung relapse was observed in 24 patients (37\%), in 19 out of field. Mediastinal lymph nodal recurrence was evidenced in 8 patients (12.3\%), 3 in field and 5 out of field.

We observed the following acute effects during radiotherapy: 30 patients with lung toxicity (grade 1-2), 24 patients with dysphagia (grade 1-2) and 12 patients with anemia (grade 1). Late heart toxicity was observed in 3 cases (4.6\%): 2 patients died for acute myocardial infarction and 1 for chronic heart failure.

We observed a significant effect of the number of lymph nodes $(\mathrm{N})$ in terms of OS $(\mathrm{p}<0.01)$ (Figure 3$)$ and DFS $(p<0.001)$ (Figure 4). Specifically, median OS

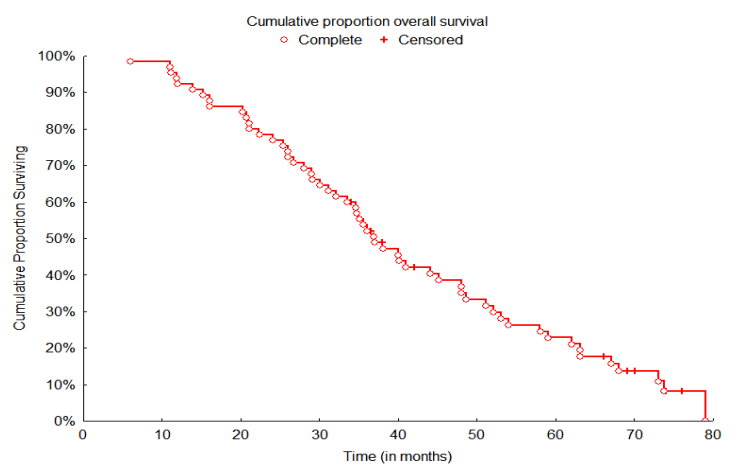

Figure 1. Median OS in Patients Stage III N2 Disease

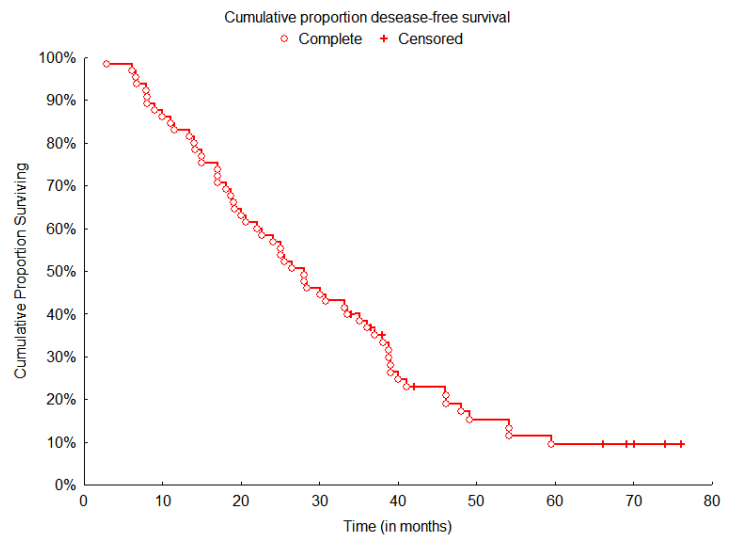

Figure 2. Median DFS in Patients Stage III N2 Disease

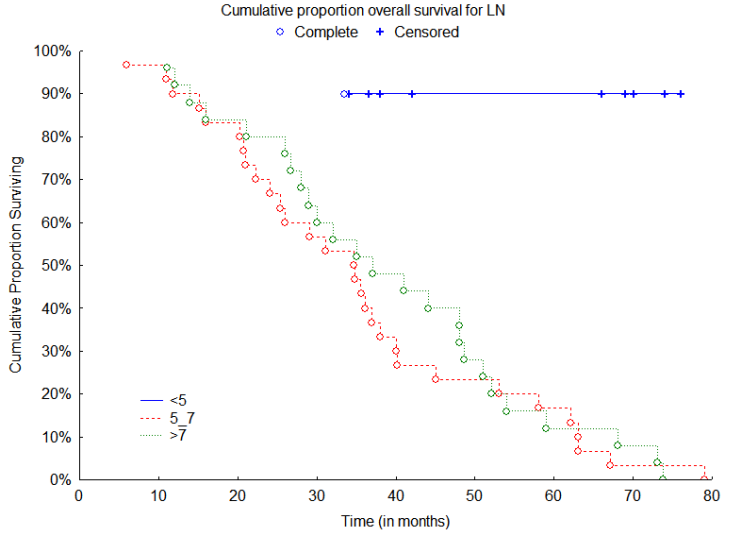

Figure 3. Cumulative Proportion of OS for Nodal Number $(\mathbf{p}<0.01)$

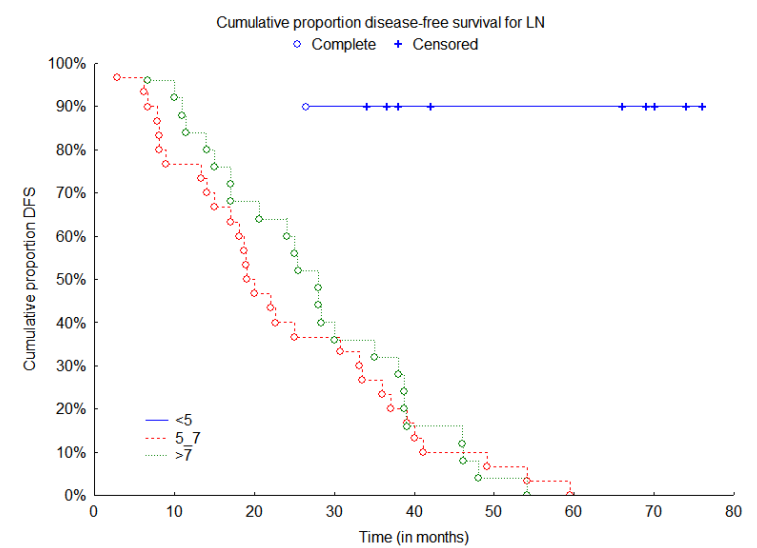

Figure 4. Cumulative Proportion of DFS for Nodal Number $(\mathbf{p}<0.001)$

and DFS for the group with $<5 \mathrm{~N}$ were 54.07 and 54.06 months respectively, for the group with 5-7 $\mathrm{N}$ were 19.57 and 34.72 months respectively, and for the group with more than $7 \mathrm{~N}$ were 27.99 and 37.04 months respectively.

Moreover, two-sample log-rank tests revealed longer survival time for patients with $<5$ lymph nodes than for patients with 5-7 nodes $(\mathrm{p}<0.001)$ and with more than 7 $(\mathrm{p}<0.001)$ nodes.

\section{Discussion}

Currently lymph node status is regarded as a valid risk stratification tool and the most powerful prognostic factor for patients with lung cancer (Osarogiagbon, 2012). However $44 \%$ of patients with pathological node negative (pN0) disease still die within 5 years. The number of stations of $\mathrm{N} 2$ nodes involved has been considered one of the most important prognostic factors for lung cancer. Though the improvement of the rate of preoperative detection of occult N2 involvement (e.g. with PET scan), many patients are still diagnosed as pN2 after surgery and then selected for multimodality treatment (Saji, 2011).

In this study we analyzed the number of mediastinal lymph nodes as predictors of survival in patients with stage III N2 NSCLC after multimodality treatment.

Recent studies showed that mediastinal nodal involvement independently influences the prognosis of 
NSCLC patients, particularly in the case of chest wall invasion (Doddoli et al., 2005; Wakelee et al., 2005; Lee et al. 2012; Mantovani et al., 2012).

Although progress has been made in treatment for locally advanced NSCLC, patients with N2 have a poor outcome even when surgical resection is complete. Currently TNM classification considers the anatomic location for every $\mathrm{N}$ category and patients with $\mathrm{N} 2$ are included in a very heterogeneous population with multiple subgroups, ranging from microscopic N2 to bulky multistation nodal involvement. Five-year survival rate is very low, from $35 \%$ for favourable N2 disease (single lymph node or single-station involvement or microscopic metastases) to $<5 \%$ in multistation, bulky N2 disease (Moretti et al., 2009). Many studies have evaluated the validity of various prognostic factors among pN2 NSCLC patients in order to identify a more accurate classification system (Nwogu et al., 2012; Macia et al., 2013). The prognosis of N2 NSCLC remaines poor and most oncologists believe that surgery alone is not sufficient for N2 disease. Many patients with N2 disease receive perioperative chemotherapy to improve their survival. It was suggested that some cases can be treat with surgery alone while others should be considered for more intensive treatments.

A recent meta meta-analysis of cisplatin-based adjuvant chemotherapy for NSCLC showed that the 5 -year survival benefit in favour of chemotherapy was $5.3 \%$ (Bria et al., 2009).

Though some studies have shown that a single station of mediastinal node metastasis was an acceptable prognostic predictor (Doddoli et al., 2005), an increased number of positive nodes was associated with a worse prognosis (Fukui et al., 2006).

The current TNM classification system for lung cancer considers only the anatomical lymph nodal extent to define the $\mathrm{N}$ category (Detterbeck et al., 2009 ;Goldstraw, 2009). However the TNM classification for other type of cancers has been updated to include the number of nodal stations involved, that has been shown to be a

more effective prognostic indicator than the anatomical location also in NSCLC (Detterbeck et al., 2009; Saji et al., 2011; Nwogu et al., 2012). It was suggested that a combined anatomically based $\mathrm{pN}$ stage classification and numerically based $\mathrm{nN}$ stage classification could be a more accurate prognostic determinant in patients with heterogeneous pN2 NSCLC (Saji et al., 2013). Thus we evaluated the number of mediastinal lymph nodes involved as predictor of survival.

Despite the limitations of this retrospective study, mainly represented by the small sample of patients and the unbalanced number of patients for each group, we confirmed that the number of lymph nodes involved has a prognostic value, related to OS and DFS: a lower number of lymph nodes is associated with a better prognosis, considering both OS and DFS, particularly in that group with a number of nodal stations involved up to 5 .

Furthermore recent studies support the role of PORT in NSCLC in terms of locoregional control and overall survival benefit (Maximus et al., 2012). Our study confirmed these data with limited toxicity during and after radiation treatment.

For patients with locally advanced disease, ongoing research is focused on appropriately identifying patients who will most benefit from the addition of surgery to a multimodality regime and safely integrating resection with chemotherapy and radiotherapy.

A multimodal strategy with surgery followed by chemotherapy and RT is a safe and reasonable treatment in locally advanced NSCLC, but no clear evidence of prognostic improvement may be assumed at the present time and in this subset of patients adequate selection of the optimal therapeutic strategy remains to be defined.

In conclusion, though the possibility of the use of multimodal strategy, such in our sample, the number of mediastinal lymph nodes involved showed to determine a poor prognosis in locally advanced NSCLC.

Studies are needed to validate the number of mediastinal lymph nodes involved as prognostic factor, in the context of an upcoming revision of TNM classification system in NSCLC.

Further investigation of combined modality treatment is warranted to improve survival in the subset of stage III N2 NSCLC

\section{Acknowledgements}

ST and CB participated in writing up and revising the manuscript. VG, BR, AP and RC analyzed the patient data and revised the manuscript. LS performed the statistical analysis. NR and VT revised the final manuscript.

All the authors read and approved the final manuscript.

\section{References}

Bradley JD, Paulus R, Graham MV, et al, Radiation Therapy Oncology Group, (2005). Phase II trial of postoperative adjuvant paclitaxel/carboplatin and thoracic radiotherapy in resected stage II and IIIA non-small-cell lung cancer: promising long-term results of the Radiation Therapy Oncology Group--RTOG 9705. J Clin Oncol, 23, 3480-7.

Bria E, Milella M, Sperduti I, et al, (2009). A novel clinical prognostic score incorporating the number of resected lymph-nodes to predict recurrence and survival in non-smallcell lung cancer. Lung Cancer, 66, 365-71.

Detterbeck FC, Boffa DJ, Tanoue LT, (2009). The new lung cancer staging system. Chest, 136, 260-71.

Doddoli C, D'Journo B, Le Pimpec-Barthes F, et al, (2005). Lung cancer invading the chest wall: a plea for en-bloc resection but the need for new treatment strategies. Ann Thorac Surg, 80, 2032-40.

End A. (2006). Diagnosis and treatment of lung cancer- nonsmall cell lung cancer, small cell lung cancer and carcinoids. Eur Surg, 38,45-53.

Fukui T, Mori S, Yokoi K, et al, (2006). Significance of the number of positive lymph nodes in resected non-small cell lung cancer. J Thorac Oncol, 1, 120-5.

Goldstraw P, (2009). The $7^{\text {th }}$ Edition of TNM in Lung Cancer: What Now? J Thorac Oncol, 4, 671-673.

Kligerman S, Abbott G, (2010). A radiologic review of the new TNM classification for lung cancer. Am J Radiology, 194, 562-573.

Lee CY, Byun CS, Lee JG, et al, (2012). The prognostic factors of resected non-small cell lung cancer with chest wall invasion. World J Surg Oncol, 10, 9. 


\section{Silvia Takanen et al}

Macia I, Ramos R, Moya J, et al, (2013). Survival of patients with non-small cell lung cancer according to lymph node disease: single pN1 vs multiple pN1 vs single unsuspected pN2. Ann Surg Oncol, 20, 2413-8.

Mantovani C, Levra NG, Filippi AR, et al, (2012). Postoperative radiotherapy for patients with completely resecate pathologic N2 non-small cell lung cancer: a retrospective analysis. Clin Lung Cancer, 9, 1-6.

Maximus S, Nguyen DV, Mu Y, et al, (2012). Size of stage IIIA primary lung cancers and survival: a Surveillance, Epidemiology and End Results Database analysis. Am Surg, 78, 1232-7.

Moretti L, Yu DS, Chen H, et al (2009). Prognostic factors for resected non-small cell lung cancer with $\mathrm{pN} 2$ status: implications for use of postoperative radiotherapy. Oncologist, 14, 1106-15.

Nwogu CE, Groman A, Fahey D, et al, (2012). Number of lymph nodes and metastatic lymph node ratio are associated with survival in lung cancer. Ann Thorac Surg, 93, 1614-9.

Okamoto L, (2008). Overview of chemoradiation clinical trials for locally advanced non-small cell lung cancer in Japan. Int J Clin Oncol, 13, 112-6.

Osarogiagbon RU, (2012). Predicting survival of patients with resectable non-small cell lung cancer: Beyond TNM. $J$ Thorac Dis, 4, 214-6.

Penland SK, Socinski MA, (2004). Management of unresectable stage III non-small cell lung cancer: the role of combined chemoradiation. Semin Radiat Oncol, 14, 326-34.

Saji H, Tsuboi M, Shimada Y, et al, (2013). A proposal for combination of total number and anatomical location of involved lymph nodes for nodal classification in non-small cell lung cancer. Chest, 143, 1618-25.

Saji H, Tsuboi M, Yoshida K, et al, (2011). Prognostic impact of number of resected and involved lymph nodes at complete resection on survival in non-small cell lung cancer. J Thorac Oncol, 6, 1865-71.

Wakelee HA, Stephenson P, Keller SM, et al, (2005). Eastern Cooperative Oncology Group, Post-operative radiotherapy (PORT) or chemoradiotherapy (CPORT) following resection of stages II and IIIA non-small cell lung cancer (NSCLC) does not increase the expected risk of death from intercurrent disease (DID) in Eastern Cooperative Oncology Group (ECOG) trial E3590. Lung Cancer, 48, 389-397. 